\title{
Collocated versus Non-collocated Multivariable Control for Flexible Structure *
}

\author{
Gary J. Balas \\ Dept. of Aerospace Engineering and Mechanics \\ University of Minnesota \\ Minneapolis, MN 55455
}

\author{
John C. Doyle \\ Dept. of Electrical Engineering \\ California Institute of Technology \\ Pasadena, Ca. 91125
}

\begin{abstract}
Future space structures have many closely spaced, lightly damped natural frequencies throughout the frequency domain. To achieve desired performance objectives, a number of these modes must actively be controlled. For control, a combination of collocated and noncollocated sensors and actuators will be employed. The control designs will be formulated based on models which have inaccuracies due to unmodeled dynamics, and variations in damping levels, natural frequencies and mode shapes. Therefore, along with achieving the performance objectives, the control design must be robust to a variety of uncertainty.

This paper focuses on the benefits and limitations associated with multivariable control design using noncollocated versus collocated sensors and actuators. We address the question of whether performance is restricted due to the noncollocation of the sensors and actuators or the uncertainty associated with modeling of the flexible structures. Control laws are formulated based on models of the system and evaluated analytically and experimentally. Results of implementation of these control laws on the Caltech flexible structure are presented.
\end{abstract}

\section{Introduction}

Collocated control is often taken to be the solution to vibration attenuation in large space structures. One benefit of this approach is that single-input/single-output (SISO) control laws can be synthesized that are robust and attenuate vibration at the collocation point. Unfortunately, collocated control is limited by the placement of the sensor/actuator pairs, the amount of force the actuators are able to be exerted on the structure and its ability to achieve performance objectives at other locations, besides the point of collocation, on the structure. Noncollocated control, on the other hand, takes advantage of measuring the exact quantity at the sensor locations to be controlled, provided the sensors are placed at locations where performance is desired. Noncollocated control is constrained by the actuators having to attenuate vibration at sensor locations through a flexible structure.

Control designs using noncollocated and collocated sensors and actuators are synthesized for the Caltech flexible structure to investigate the benefits and limitations of each approach. This paper addresses the question of whether performance is restricted by the noncollocation of the sensors and actuators or the uncertainty associated with modeling of the flexible structure. These results are compared to the use of collocated sensors and actuators for vibration suppression. Both theoretical and experimental results are included.

The paper layout is as follows: Section 1 provides an introduction to the control design problem. Section 2 details the Caltech experimental flexible structure with a brief background on the structured singular value $(\mu)$ framework, used for control design, in sec- tion 3. Section 4 describes the control problem formulation with the results presented in section 5. A summary of the research is provided in the final section.

\section{Caltech Flexible Structure}

The Caltech experimental flexible structure is designed to include a number of attributes associated with large flexible space structures [BalDoy1, BalDoy2, Balas]. These include lightly damped, closely spaced modes, collocated and noncollocated sensors and actuators, and numerous modes in the controller crossover region.

Two different phases of the structure have been construeted. The Phase I structure consists of a two story, three longeron truss with three noncollocated sensors and actuators. The Phase II structure has three stories, three longerons with three collocated and three noncollocated sensors and actuators. The Phase II structure is used to examine collocated and noncollocated control issues.

The Phase II design of the Caltech flexible experiment shown in figure 1. The first story columns are $6.35 \mathrm{~mm}\left(\frac{1}{4} \mathrm{in}\right.$.) diameter aluminum rods, $.838 \mathrm{~m}(33 \mathrm{in}$.) long. The first platform is a solid equilateral triangular with a $.406 \mathrm{~m}$ (16 in.) base. The second story columns are $4.76 \mathrm{~mm}\left(\frac{3}{16} \mathrm{in}\right.$.) diameter aluminum rods, $.559 \mathrm{~m}$ (22 in.) in length, and the third story columns are $.813 \mathrm{~m}$ (32 in.) long and the same diameter as the second bay. The total height of the Phase II structure, including the platforms, is $2.32 \mathrm{~m}$ (87.88 in.). The second and third story platforms are $6.35 \mathrm{~mm}\left(\frac{1}{4} \mathrm{in}\right.$.) thick aluminum plates with the same dimensions as the first bay but with an equilateral triangle, of base dimension $.356 \mathrm{~m}$ (14 in.) cut out of their center. This reduces the weight of each bay, thereby increasing the natural frequencies of the global flexible modes. The longerons connect the stories via triangular mating fixtures and three bolts. All the longerons are shrunk fit and welded to the mating brackets. The entire structure hangs from a mounting structure fixed to the ceiling.

The three voice coil actuators act along the first bay diagonals. Three additional proof mass actuators are mounted to the bottom, third bay, and positioned parallel to the sides of the triangular platform. The center of each proof mass actuator is located in the center of the platform side. Three accelerometers are positioned parallel to the platform sides, corresponding to the proof mass actuators, and located on bays 2 and 3 . The three accelerometers on platform 3 are collocated with the proof mass actuators. The actuators are mounted on the bottom of the platform and the sensors on top. There are three additional linear voltage displacement transducers (LVDT) sensors located on the proof mass actuators. These are used in a local fedback loop to linearize the response of the proof mass actuators.

\footnotetext{
-Funding for this research was provided by the NASA Graduate Student Fellowahip, Caltech President'a Fund and the COFS Gueat Investigator Program
} 


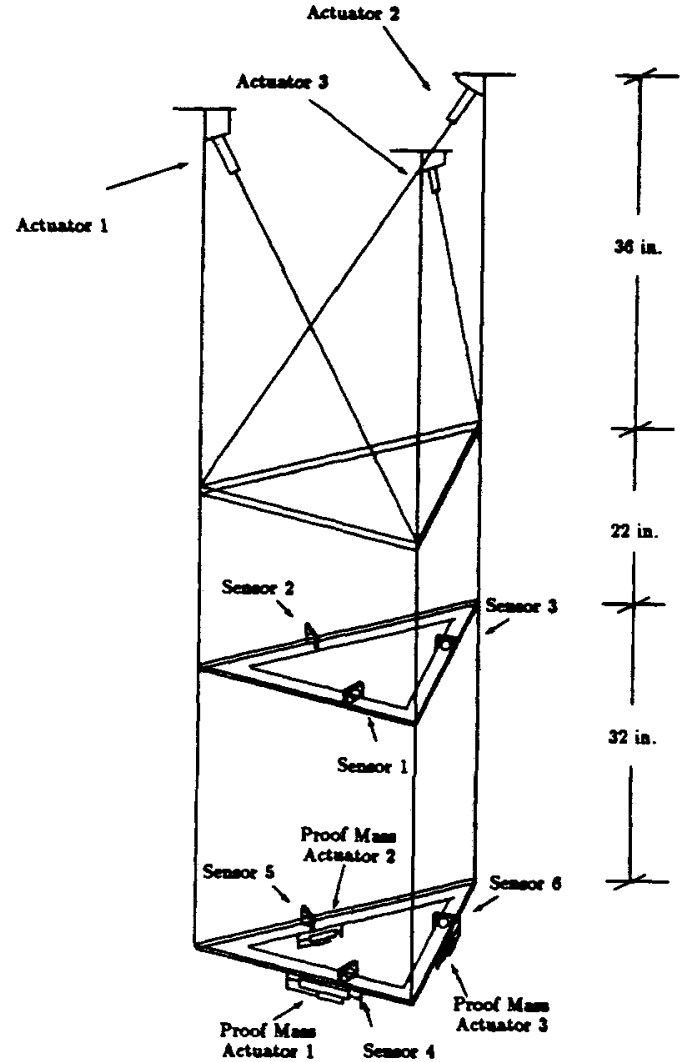

Figure 1: Phase II Caltech Flerible Structure

\subsection{Accelerometers}

The sensors are Sunstrand QA-1400 accelerometers. The accelerometers have a flat frequency response between 0 and $200 \mathrm{~Hz}$. The noise associated with them is rated at $0.05 \%$ of the output at $0.10 \mathrm{~Hz}$ and $2 \%$ at $10-100 \mathrm{~Hz}$. The sensors are scaled for accelerations of approximately $0.016 \mathrm{~g}$ per volt. This provides a maximum \pm 5 volts output at peak accelerations of the input disturbance. The accelerometer output is conditioned by a $100 \mathrm{~Hz}, 4^{\text {th }}$ order Butterworth filter prior to input into the $A / D$, providing attenuation of the high frequency signals and noise.

\subsection{Linear Voltage Displacement Transducers}

The three linear voltage displacement transducers (LVDTs) are TransTek model 244. They are designed to measure displacements up to \pm 1.5 inches accurately. The input voltage is selected such that a displacement of \pm 1.0 inch correspond to \pm 5 Volts. These transducers have zero hysteresis, and are linear across their operating range to within $\pm 0.5 \%$ of their output voltage.

\subsection{Voice Coil Actuators}

The actuators are a voice coil type design, built by Northern Magnetics Inc., that output a force proportional to an input voltage. The actuators are rated at $\pm 1.37 \mathrm{~kg}$ ( $3 \mathrm{lbs}$ ) of force at \pm 5 volts and have a bandwidth of $60 \mathrm{~Hz}$.

\subsection{Proof Mass Actuators}

The proof mass actuatorn, built by Northern Magnetics Inc., operate on the same principles as the voice coil actuators. A set of permanent magnets are mounted on a shaft, which is surrounded by a winding of wire and a plastic sleeve. The winding and plastic casing ride on bearings along the shaft holding the permanent magnet. By passing a current through the windings, a magnetic flux is generated, producing a force. The force sets the winding and sleeve in motion relative to the shaft and magnets, with the direction of the movement depending on the polarity of the current. The movement of the windings and metal casing produces an inertial force, which is transmitted to the structure.

Ideally, the voltage input would be proportional to the force output. This is not the case as the strength of the permanent magnetic draws it to the LVDT case, core and its bearings, causing alignment problems and rubbing between the mounting structure and the LVDT. This leads to stiction and friction in the actuators. A parasitic effect is noticed due to the realignment of the magnetic field in the LVDT case with each oscillation. The combination of these effects result in the actuator exhibiting a nonlinear response to input excitation. A local feedback system, employing an LVDT sensor, is designed to provide centering of the actuator at low frequencies and improved linear response in the frequency range of 0.5 Hs to $8 \mathrm{~Hz}$ [Balas].

\subsubsection{Proof Mass Actuator Control Laws}

The nonlinear response of the proof mass actuators presents a difficult control problem. At low amplitude, the command signal needs to be large to overcome the stiction. As the amplitude of the command signal increases, so does the gain of the proof mass actuator. Therefore a pure high gain fedback loop will destabilize the system as the input command amplitude is increased. As the command signal increases, the control law needs to guard against the amplitude of response so as not to exceed the force limitations on the motor.

A nonlinear control law is developed, which nonlinearly amplifies small signats and exhibits a linear relationship as the signal level increases. The linear control law has an integrator at low frequency to center the mass with phase lead to push the bandwidth of the system out to approximately $8 \mathrm{~Hz}$. The closed-loop response of the proof mass actuators varies by a factor of 2.5 between 0.2 and $8 \mathrm{~Hz}$, for input amplitudes varying between 0 and 360 . The deviation of the actuator from a linear response is accounts for in the control design by uncertainty modele.

\subsection{Modeling of Experimental Structures}

A model of the atructure relating input signals to symtem outputs is desired for control design purposes. Initially, an input/output model is developed from first principles. The model is refined for control purpoces using system identification techniques [BalDoy2].

The first nine global modes, between 0.90 and $6.3 \mathrm{~Hz}$, of the structure are of interest for control purposes. The first group of local modes occur in the frequency range of 37 to $43 \mathrm{~Hz}$. Table 1 contains.

\begin{tabular}{|c|c|c|c|c|}
\hline Mode & $\begin{array}{c}\text { NASTRAN } \\
\text { Natural } \\
\text { Frequency (Hz) }\end{array}$ & $\begin{array}{c}\text { Experimental } \\
\text { Natural } \\
\text { Frequency (Hz) }\end{array}$ & $\begin{array}{c}\text { Damping } \\
\text { Ratio }\end{array}$ & $\begin{array}{c}\text { Mode } \\
\text { Type }\end{array}$ \\
\hline 1 & 0.76 & 0.90 & $0.62 \%$ & 1st bending \\
2 & 0.76 & 0.92 & $0.82 \%$ & 1st bending \\
3 & 1.84 & 1.62 & $1.39 \%$ & 1st tonnional \\
4 & 1.73 & 2.21 & $1.21 \%$ & 2 2nd bending \\
5 & 1.73 & 2.29 & $1.22 \%$ & 2ad bending \\
6 & 3.40 & 3.60 & $1.84 \%$ & 2ad torsional \\
7 & 4.14 & 3.94 & $0.74 \%$ & 3rd bending \\
8 & 4.14 & 3.97 & $0.64 \%$ & 3rd bending \\
9 & 8.21 & 6.30 & $0.96 \%$ & 3rd torional \\
\hline
\end{tabular}

Table 1: Damping Ratios and Frequencies of the Phase II Truss 
a list of natural frequencies derived from the Nastran finite element model and the natural frequencies and damping values derived from experimental dats. Two transfer function plots of the experimental data versus the identified model for select actuators and sensors are shown in figures 5 and 7.

\section{$3 \quad \mu$-Framework}

This section briefly reviews frequency domain methods for analyzing the performance and robustness properties of feedback systems using the structured singular value $(\mu)$ [Doy1, Doy2, Pack]. The general framework, shown in figure 2 , is based on linear fractional transformations (LFTs). Any linear interconnection of inputs, outputs, and commands along with perturbations and a controller can be viewed in this context and rearranged to match this diagram. $P$ represents the system interconnection structure, $\Delta$ the uncertainties, and $K$ the control law. $v$ is a vector of exogenous inputs and disturbances, $e$ is a vector of errors to be kept small, $y$ is a vector of measurement signals provided to the control design, $u$ is a vector of inputs from the control law, $z$ and $\bar{u}$ are outputs to and from the uncertainty block.

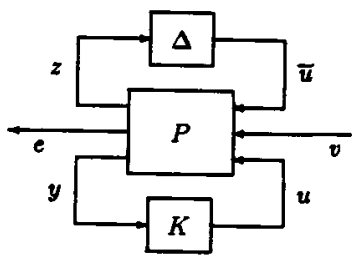

Figure 2: General Interconnection Structure

\subsection{Analysis Overview}

For the purpose of analysis, the controller may be thought of as just another system component. The inclusion of the controller into the plant reduces the diagram in figure 2 to that in figure 3-(a). The analysis problem involves determining whether the error $e$ remains in a desired set for sets of input $v$ and perturbation $\Delta$. The uncertainty in $v$ and $\Delta$ as well as the performance specifications on $e$ are normalized to 1 . This requires that all weighting functions and scalings be abeorbed into the interconnection structure $\boldsymbol{G}$. Furthermore, $G$ can be partitioned so that the input-output map from $v$ to $e$ can be expressed as the following linear fractional transformation

$e=F_{u}(G, \Delta) v \quad$ where $F_{u}(G, \Delta)=G_{22}+G_{21} \Delta\left(I-G_{11} \Delta\right)^{-1} G_{12}$

Robust stability and robust performance questions associated with the interconnection structure $G$ are characterized by a structured singular value $(\mu)$ test of the system in the presences of structured uncertainty.

\subsection{Synthesis Review - $H_{\infty}$ Optimization}

For the purpose of synthesis, the $\Delta$ can be normalized properly to 1 so that the normalizing factor can be absorbed into $P$. This
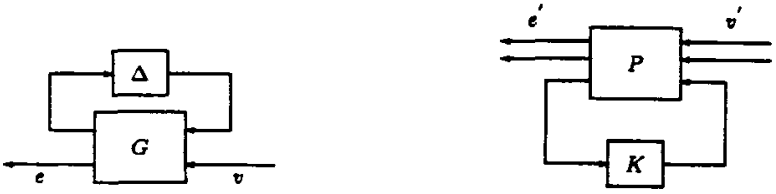

Figure 3: (a) Analysis

and

(b) Syntheais Problem results in the synthesis problem as shown in figure 3-(b). Hence, the synthesis problem involves finding a stabilizing controller $K$ such that the performance requirements are satisfied under prescribed uncertainties. The interconnection structure $P$ can be partitioned so that the input-output map from $v^{\prime}$ to $e^{\prime}$ can also be expressed as the following linear fractional transformation

$e^{\prime}=F_{7}(P, K) v^{\prime} \quad$ where $\quad F_{7}(P, K)=P_{11}+P_{12} K\left(I-P_{22} K\right)^{-1} P_{21}$

For the $H_{\infty}$ optimal control problem, the objective is to find a stobilizing controller $K$ which minimizes $\left\|F_{l}(P, K)\right\|_{\infty}$. A detailed discussion of recent state-space results are given in [DGKF, GlovDoy1].

\section{$3.3 \mu$-Synthesis Methodology}

The $\mu$-synthesis methodology is an approach to designing control systems with robust performance. This technique entially integrates two powerful theories for synthesis and analysis into a sys tematic design technique involving $H_{\infty}$ optimization methods for synthesis and the structured singular value $(\mu)$ for analysis.

$A$ bound on $\mu$ may be obtained by scaling and applying the $\|\cdot\|_{\infty}$. Extending this concept to synthesis, the problem of robust controller design becomes that of finding a stabilising controller $K$ and scaling matrix $D$ such that the quantity $\left\|D F_{l}(P, K) D^{-1}\right\|_{\infty}$ is minimized. One approach for solving this problem is that of alternately minimizing the above expression for either $K$ or $D$ while holding the other constant. For fixed $D$, it becomes an $H_{\infty}$ optimal control problem and can be solved using the well-known state-space method. On the other hand, with fixed $K$, the above quantity can be minimized at each frequency as a convex optimization in $\ln (D)$. The resulting data of $D$ can be fit with an invertible, stable, minimumphase, real-rational transfer function. This process is carried out iteratively until a satisfactory controller is constructed.

The $\mu$-synthesis techniques have been used extensively in the area of designing robust control laws for flexible structures. Control designs using this methodology have been formulated for the vibration attenuation problem and implemented on a number of flexible structures experiments [Balas, BalChuD, BalDoy2]. The control designs synthesized using $\mu$-synthesis achieved a high level of vibration attenuation along with good robustness characteristics. The $\mu$-synthesis design methodology is employed to address the benefits and limitations of collocated versus noncollocated control design for flexible structures.

\section{Noncollocated Versus Collocated Con- trol Design}

Control designs using noncollocated and collocated sensors and actuators are synthesized for the Phase II flexible structure. The first design uses the voice coil actuators to attenuate vibration of the third story of the structure, which is excited by proof mass actuator 1. Proof mass actuator 1 is collocated with sensor 4. This design shows the benefit of measuring the signals to be controlled and limitations of acting through a flexible structure. The second design employs the proof mass actuators to attenuate vibration at collocated sensors. The disturbance to the structure enters through voice coil actuator 1 . The achievable performance of the proof mass actuators is seen to be constrained by the force it is able to impart 
on the atructure and by the linearity of the actuators. The collocated design is only able to attenuate vibration of the structure locally.

\subsection{Control Objective}

The performance objective is to attenuate the vibration of sensors 4,5 , and 6 mounted to the third story platform. A diagram of the Phase II structure and sensor locations is provided in figure 1. The disturbance enters at proof mass actuator 1 , which is collocated with sensor 4, for the noncollocated design with the voice coil actuators used for control. The collocated control design is excited by voice coil actuator 1 with the proof mass actuators used for control. The input disturbance signal has all its energy concentrated between 0.2 and $12.7 \mathrm{~Hz}$, with a one pole roll off at $1 \mathrm{~Hz}$. The performance criteria is defined as minimizing the $\|\cdot\|_{\infty}$ norm of the transfer function from the input disturbance to sensors 4,5 , and 6 .

\subsection{Uncertainty Descriptions}

An additive uncertainty is included in the problem formulation and performs thre functions: it accounts for the unmodeled high frequency modes, limits the control bandwidth and describes model errors inside the control bandwidth. The additive uncertainty weight for the noncollocated control design, using the voice coil actuators for control, is given by $W_{\text {edditive-1 }}=5 \frac{(1+25)^{2}}{(0+250)^{2}}$

The collocated control design employs the proof mass actuators for control. In spite of the local feedback loop on the proof mass actuators, there is considerable variation in the output displacement of the actuators above $5 \mathrm{~Hz}$. The additive uncertainty weight is selected to limit the control bandwidth to $7 \mathrm{~Hz}$ to limit high frequency excitation. The weighting function is given by $W_{\text {additiven }}=$ $1.2 \frac{(1+25)^{2}}{(1+500)^{2}}$

\subsection{Problem Formulation}

Control designs are formulated using the $\mu$-8ynthesis methodology to address the issue of noncollocated versus collocated control design. Both control designs employ additive uncertainty to account for model error. The voice coil (VC) actuators are used for control purposes in the noncollocated problem formulation. The magnitude of the VC actuators is constrained to $\pm 3 \mathrm{lb}$ of force for $\pm 5 \mathrm{~V}$ input signals included in the noncollocated problem formulation. The local feedback loop around the proof mass actuators used in the collocated control design requires the control laws to command displacement of the moving mass. The stroke limit on the moving mass, \pm 1.0 in., is translated into a magnitude bound of 1500 on the proof mass actuator commands. These constraints on the actuators are included in the problem formulation as magwt.

The input disturbance in both designs is modeled as a white noise signal filtered by $\frac{6.2}{i+6.2}$. The $\|\cdot\|_{\infty}$ norm of the transfer function between the disturbance input to the sensor output represents the performance objective in the control problem formulation.

\subsubsection{Noncollocated}

In the noncollocated control design problem, the transfer function between the input disturbance at proof mass actuator 1 and sensor 4 rolls up like an $8^{2}$ response between 1 and $8 \mathrm{~Hz}$. This is seen in figure 7. Based on the initial control designs, the voice coil actuators are only able to affect the flexible modes of the strueture. Therefore, only attenuation of the resonant peaks is specified in the performance criteria. Sensors 4,5 , and 6 are filtered by $\frac{235}{(s+15)^{2}}$ to weigh the resonant peales of the structure equally and scale them accordingly for performance. These are defined by perfwt in the control problem block diagram. For performance, an 8 to 1 reduction in the peaks of the flexible mode is desired. A sensor noise level of $2 \times 10^{-3}$ is included in the design. The act $u$ weight is selected to be .1 which translates into a $1 \%$ input multiplicative uncertainty.

\subsubsection{Collocated}

The resonant peaks of the transfer function between voice coil actuator 1 and sensors 4,5 , and 6 , seen in figure 5 , are initially scaled to one. A constant performance weight, perfut in the block diagram, is used to select the desired vibration attenuation level. For performance, an 10 to 1 reduction in the peaks of the flexible mode is desired. A sensor noise level of $2 \times 10^{-3}$ is included in the design. The act $u$ weight is selected to be .1 which translates into a $1 \%$ input multiplicative uncertainty.

\subsection{Control Designs}

There are a limited number of weights used in the noncollocated and collocated control problem formulations. This is due to the nominal models, which include disturbance excitations, having 35 states. The addition of the additive uncertainty weight, input disturbance filter, and performance weight, leads to the design model with 50 states. A block diagram of the problem formulation is given in figure 4. This diagram is reformulated into the general LFT framework for the application of $\mu$-synthesis techniques.

The proof mass actuator local control loop is always turned on during the open-loop and closed-loop experiments. In the open-loop configuration, the local loops keep the moving mass centered. Experimental frequency responses are determined with the local loop on and off with the moving masses fixed to examine the effect of the proof mass actuator local feedback loops. Turning on the local feedback loops adds damping to the torsional modes of the structure. The local feedback loop increased damping levels by a factor of 2.5 on several of the torsional modes. A detriment in using the local proof mass control loops is that they increase the level of ambient disturbance above $7 \mathrm{~Hz}$ by a factor of 10 . This is attributed to the stiction/friction in the proof mass actuators vibrating the structure.

\section{Results}

The behavior of the proof mass actuators limits performance of the control designs for both the collocated and noncollocated case. The proof mass actuators impart large forces at high frequency which translate into large accelerations of the collocated sensors. These accelerations are on the order of the response of the structure due to excitation of a flexible mode, shown in figure 7 . The voice coil actuators are only able to substantially affect the flexible modes of the structure. Therefore, only minimal vibration attenuation is possible at frequencies that are not associated with flexible modes regardless of the accuracy of the model.

The achievable vibration attenuation using the proof mass actuators as control actuators is limited. The control authority of these actuators is hindered at low frequency by low force levels. The inertial force transmitted to the structure is a function of the moving mass and its acceleration. Acceleration is the second derivative of displacement, hence to achieve the same force at half the natural frequency, the moving mass requires four times the displacement. 


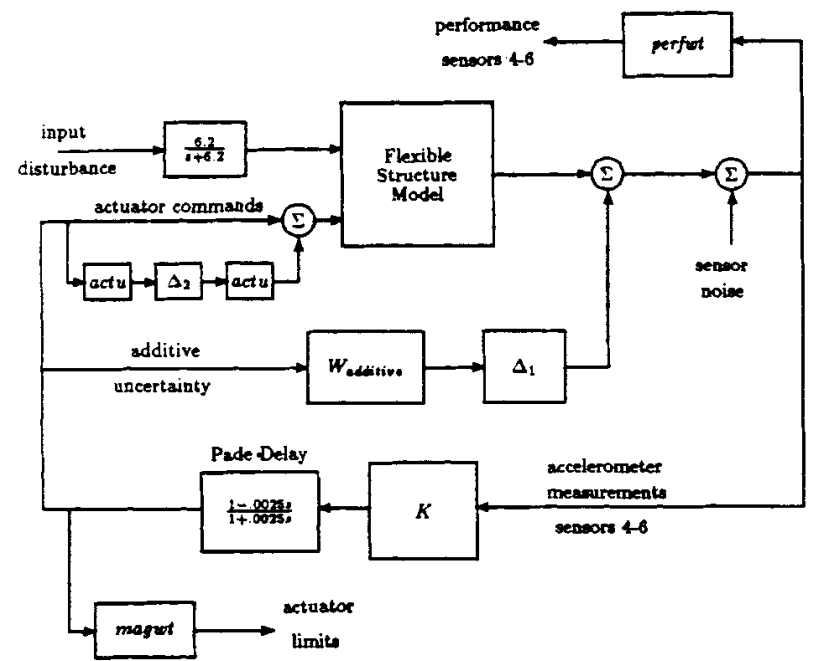

Figure 4: Block Diagram of the Noncollocated/Collocated Control Problem

Given that the moving mass is approximately $0.35 \mathrm{~kg}(0.8 \mathrm{lb})$ and the maximum stroke is \pm 1.0 in., the proof mass actuator generates $4 \times 10^{-3} \mathrm{lb}$ of force at the first natural frequency of the structure, $0.9 \mathrm{~Hz}$. This is contrasted by the ability of the voice coil actuators to input up to $3 \mathrm{lb}$ of force at this frequency.

The effects of stiction/friction are noticed in the response of the proof mass actuators despite the local feedback loop. The local feedback around the proof mass actuators does not completely eliminate the problems due to the stiction/friction. The output force of proof mass varies considerably above $5 \mathrm{~Hz}$. This is a severe limitation when the proof mass actuators are used for control purposes. This is especially evident at the third torsional mode of the structure. The high frequency forces impacted to the structure from the proof mass actuators limit the performance of the collocated control laws.

The noncollocated control design emphasized attenuating the response of the nine flexible modes of the Phase II experiment between 0.9 and $6.3 \mathrm{~Hz}$, and has minimal or no effect at other frequency points. One can infer from the loop gain of the control design, that the first and second bending and torsional modes will be heavily attenuated and the third bending and torsional modes will have little attenuation. Analyzing the noncollocated design using $\mu$ indicates that the control design can not meet the performance or robustness specifications in the frequency range 3.2 to $20 \mathrm{~Hz}$. Experimental results confirm these obeervations. Figures 5 is experimental transfer function plot of the open-loop and closed-loop system at sensor 4 with the noncollocated control law, $K 5 v$, implemented. The control design attenuates the resonance peaks of the first six flexible modes by at least a factor of 4 . Above $5 \mathrm{~Hz}$, the input disturbance swamps the control authority of the voice coil actuators.

The noncollocated control laws employ the voice coil actuators to achieve the performance objectives by attenuating the vibration of the entire structure. This is seen by the attenuation of the frequency peaks at sensor 1 in figure 6 . The only mode not attenuated is the third bending mode at $3.97 \mathrm{~Hz}$, due to its small response at the third story sensors.

The collocated control designs attenuate vibration at the sensors by limiting the motion of the third platform. The entire structure may be vibrating provided the platiorm remains quiet. The proof mass actuators are able to attenuate vibration of the torsional modes the best. This is expected because they exert force perpendicular to a lever arm from the center of the structure. Unfortunately, these actuators are of less assistance in attenuating vibration of the second and third bending modes. The proof mass actuators have little control authority over the response of these modes while the voice coil actuators have their largest influence on these modes.

The performance objective is the same as in the noncollocated design. Notice that the proof mass actuator control laws attenuate vibration only at sensors 4,5 and 6 . Figure 8 is a comparison of the open-loop and closed-loop response of sensor 1 with the collocated control law. The first bending and torsional modes are heavily attenuated since their response is coupled to the response of sensors 4, 5 and 6. The higher modes exhibit the same level of response open-loop and closed-loop. Collocated control laws provide minimal vibration attenuation at other locations on the structure not coupled to the collocated sensors. Performance of the collocated designs at sensors 4,5 , and 6 would improve provided the model error is reduced but this would not reduce the response of sensors 1,2 , and 3 due to the excitation.

\section{Conclusions}

Clearly, the performance requirements and locations of the actuotors and sensors will dictate the use of collocated or noncollocated control laws. The control engineer needs to understand the advantages and disadvantages of each approach and how model error effects the design problem in order to make an intelligent selection of a control strategy. Hopefully, the results presented in this paper provide insight into the benefits and limitations of each approach to vibration attenuation.

\section{References}

[Balas] G.J. Balas, "Robust Control of FLexible Structures: Theory and Experiments," Ph.D. Thesis, California Institute of Technology, Pasadena, 1989.

[BalChuD] G.J. Balas, C.C. Chu and J.C. Doyle, "Vibration Damping and Robust Control of the JPL/AFAL Experiment Using $\mu$-Synthesis," Proceedings of $28^{\text {th }}$ Conference on Decision and Control, Ft. Lauderdale, FL, December, 1989.

[BalDoy1] G.J. Balas and J.C. Doyle, "Robust Control of Flexible Modes in the Controller Crossover Region," ACC89, Pittsburgh, PA, 1989.

[BalDoy2] G.J. Balas and J.C. Doyle, "Identification for robust control of flexible structures," to appear Control System Magazine, March, 1990.

[DGKF] J.C. Doyle, K. Glover, P. Khargonekar and B.A. Francis. "State-space solutions to standard $H_{2}$ and $H_{\infty}$ control problems," IEEE Transactions on Automatic Control, Vol. 34, No. 8, August, 1989, pp 831-847.

[Doy1] J.C. Doyle, "Analysis of feedback systems with structured uncertainties," Proc. IEE-D 129, 1982, pp 242-250.

[Doy2] J.C. Doyle, Lecture notes on edvances in multiveriable control, ONR/Honeywell Workshop on Advances in Multivariable Control, Minneapolis, MN, October, 1984.

[GlovDoy1] K. Glover and J.C. Doyle, "State-space formulae for all stabilizing controllers that satisfy an $H_{\infty}$-norm bound and relations to risk sensitivity," Systems \& Control Letters 11, 1989, pp 167-172. 
[Pack] A.K. Packard, "What's new with $\mu$ : structured uncertainty in multivariable control," Ph.D. Thesis, University of California at Berkeley, 1988.

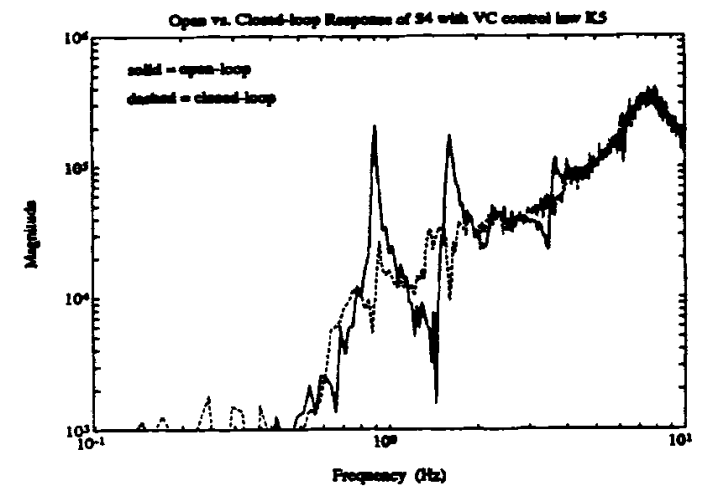

Figure 5: Opea ra. Clowed-boop Respocee of S4 with the Noseolbeated Control Lan

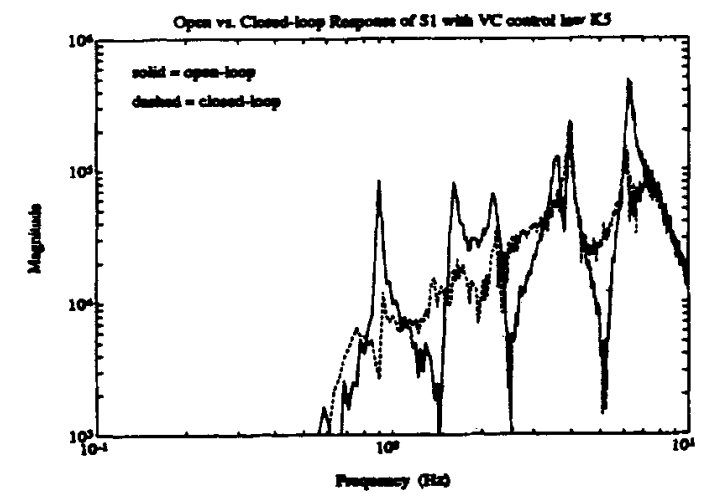

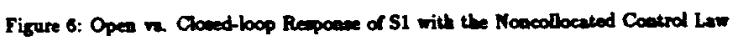

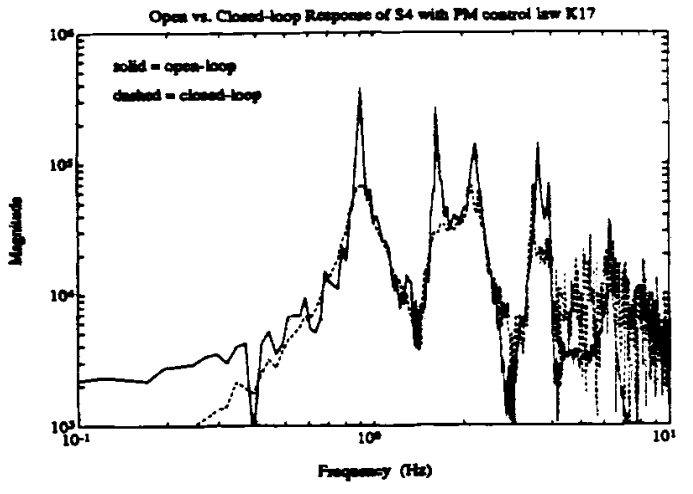

Figure 7: Opea n. Cloced-loop Response of S4 with the Collocated Control Law

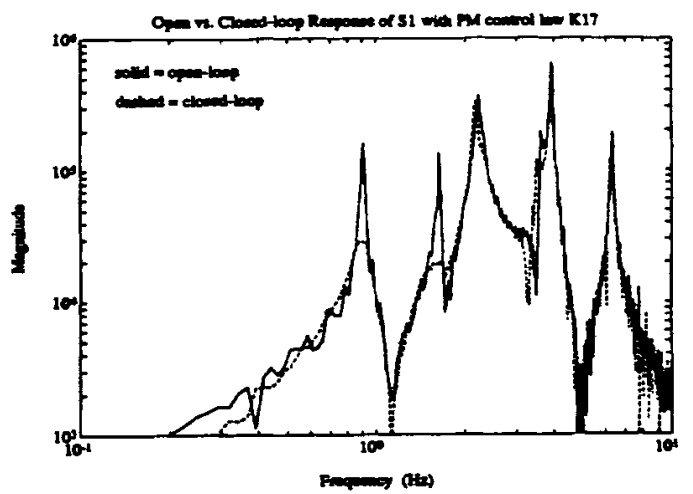

Figre 8: Opea n. Cloned-loop Repoene of S1 with the Colbated Control Law 